\title{
Increased Plasma Levels of Nesfatin-1 in Patients with Newly Diagnosed Type 2 Diabetes Mellitus
}

\author{
Authors \\ Z. Zhang ${ }^{1 *}$, L. Li ${ }^{2 *}$, M. Yang ${ }^{1}$, H. Liu ${ }^{3}$, G. Boden ${ }^{4}$, G. Yang ${ }^{1}$ \\ Affiliations \\ Affiliation addresses are listed at the end of the article
}

Key words

- insulin resistance

- type 2 diabetes mellitus

- nesfatin-1 received $\quad 23.06 .2011$

first decision 09.08.2011

accepted 24.08.2011

\section{Bibliography}

DOI http://dx.doi.org/

10.1055/s-0031-1286339

Published online:

October 21, 2011

Exp Clin Endocrinol Diabetes

2012; 120: 91-95

(C) J. A. Barth Verlag in

Georg Thieme Verlag KG

Stuttgart · New York

ISSN 0947-7349

Correspondence

Gangyi Yang

Department of Endocrinology

the Second Affiliated Hospital

Chongqing Medical University

Tel.: +86/23/684 86115

Fax: $+86 / 23 / 68486115$

gangyiyang@yahoo.com.cn

\section{Abstract}

$\nabla$

Background: Nesfatin-1, which is derived from nucleobindin2 (NUCB2), has been recently identified as a novel satiety regulator. However, its pathophysiological role in humans remains unknown. The aim of the present study was to investigate plasma nesfatin-1 levels and the association between plasma nesfatin-1 levels and various metabolic parameters in humans.

Materials and methods: 74 subjects with newly diagnosed type 2 diabetes mellitus (nT2DM), 73 subjects with impaired glucose tolerance (IGT) and 73 subjects with normal glucose tolerance (NGT) were enrolled in this study. Plasma nesfatin-1 levels were measured by a commercially available enzyme- linked immunosorbent assay. Results: Plasma nesfatin-1 levels were elevated in subjects with both nT2DM and IGT compared

\section{Introduction}

$\nabla$

Nesfatin-1, derived from the nucleobindin2 (NUCB2)precursor, contains 82 amino acids and is highly conserved in humans, rats and mice. It has been reported that nesfatin-1 suppresses nocturnal food intake and reduces body weight gain when injected into the third ventricle of rodents, whereas infusion of NUCB2 antisense oligonucleotide stimulates food intake (Oh-I S et al., 2006; Johnstone et al., 2006; Stengel et al., 2009). Furthermore, recent studies have shown that nesfatin-1 reduces food intake in rodents when administered peripherally (Shimizu et al., 2009). Plasma levels of nesfatin-1 are reduced by fasting, and increased after refeeding (Kohno et al., 2008). This suggests that nesfatin- 1 plays a role in satiety regulation and, possibly, energy homeostasis (Kohno et al., 2008).

\footnotetext{
${ }^{*}$ These authors contributed equally to this project.
}

to controls $(1.91 \pm 0.79$ and $1.80 \pm 0.80$ vs. $1.41 \pm$ $0.58 \mu \mathrm{g} / \mathrm{L}, P<0.05$ or $P<0.01$ ). Simple regression analysis showed that in subjects with IGT and nT2DM, plasma nesfatin-1 correlated positively with body mass index (BMI), hemoglobin $\mathrm{A}_{1} \mathrm{C}$ $\left(\mathrm{HbA}_{1 \mathrm{c}}\right)$, fasting blood glucose (FBG), $2 \mathrm{~h}$ blood glucose after a glucose load (2hPBG), fasting plasma insulin (FINS) and the homeostasis model assessment of insulin resistance (HOMA- ${ }_{I R}$ ). Multivariate logistic regression analysis revealed that plasma nesfatin-1 was significantly associated with IGT and nT2DM, even after controlling for differences in BMI.

Conclusion: Plasma nesfatin-1 concentrations were found to be elevated in subjects with both IGT and nT2DM and to be related with several clinical parameters known to be associated with insulin resistance.
The nesfatin- 1 is prominently expressed in several regions of the hypothalamus and spinal cord autonomic nuclei. Also, it has been demonstrated to be present in peripheral tissues including adipocytes, gastric mucosa and in human and rat pancreatic beta-cells, indicating the possible involvement of nesfatin- 1 in the regulation of insulin secretion from pancreatic beta-cells (Stengel et al., 2009; Goebel et al., 2009; Foo et al., 2010).

Obesity is mainly caused by excessive food intake and/or reduced energy expenditure. It is an important public health problem and is associated with insulin resistance and T2DM (Rasouli and Kern, 2008). As nesfatin-1 may play an important role in the regulation of body weight and insulin secretion, we hypothesized that plasma nesfatin-1 levels might be affected by insulin resistance in subjects with newly diagnosed type 2 diabetes mellitus (nT2DM) and impaired glucose tolerance (IGT). We, therefore, 
measured plasma nesfatin-1 levels in these groups and correlated them with anthropometric and metabolic parameters.

\section{Materials and Methods}

\section{Study patients}

220 Chinese volunteers were involved in this study and categorized into 3 groups.

74 patients with nT2MD (39 men and 35 women; age $54 \pm 11$ years; body mass index (BMI), $25.0 \pm 3.7 \mathrm{~kg} / \mathrm{m}^{2}, \mathrm{nT} 2 \mathrm{MD}$ group) and 73 subjects with IGT ( 35 men and 38 women; age $54 \pm 10$ years; BMI, $24.7 \pm 2.7 \mathrm{~kg} / \mathrm{m}^{2}$, IGT group) were recruited in the study. T2DM and IGT were diagnosed with a $75 \mathrm{~g}$ oral glucose tolerance test (OGTT) according to Word Health Organization criteria (Alberti and Zimmet, 1998). The patients with IGT and T2DM were newly diagnosed and had not previously received any diabetic medications or diets. 73 age-and BMI-matched healthy subjects who had normal glucose tolerance (36 men and 37 women; age $51 \pm 7$ years; BMI, $24.5 \pm 3.6 \mathrm{~kg} / \mathrm{m}^{2}$, NGT group) were selected as controls. None of healthy subjects had a family history of diabetes or other endocrine disorders or were taking any medication known to alter glucose tolerance. Their body weight was stable for at least 2 months. Patients with type 1 diabetes mellitus, acute or chronic infectious disease, pregnancy, heart failure, hypertension, liver or kidney disease and cancer were excluded from the study. All study subjects were of Han Chinese origin. This study was approved by the Ethics Committee of Chongqing Medical University, and informed consent was obtained from all study participants.

\section{Anthropometry and blood samples}

Anthropometric measurement was performed in the morning, before breakfast, with subject wearing light clothing, without shoes. Body weight and height were measured in all subjects using a scale and a wall-mounted stadiometer to the nearest $0.5 \mathrm{~kg}$ and $0.5 \mathrm{~cm}$ respectively. Waist and hip circumferences were measured using standard techniques. The waist to hip ratio (WHR) was calculated as the ratio of waist and hip circumferences. A $75 \mathrm{~g}$ oral glucose tolerance test was conducted after a $12 \mathrm{~h}$ overnight fast. Body mass index (BMI)was calculated as the body weight in kilograms divided by the height in meters squared. The homeostasis model assessment of insulin resistance (HOMA-IR) and insulin secretion (HOMA-IS)were calculated using the following equations (Albareda et al., 2000): $\mathrm{HOMA}_{-\mathrm{IR}}=$ fasting insulin $(\mu \mathrm{U} / \mathrm{ml}) \times$ fasting glucose $(\mathrm{mmol} / \mathrm{L}) / 22.5$, and $\mathrm{HOMA}_{\mathrm{IS}}=[20 \times$ fasting insulin $(\mu \mathrm{U} / \mathrm{ml})] /[$ fasting blood glucose $(\mathrm{mmol} / \mathrm{L})-3.5]$.

Venous blood samples were obtained at 0, 30, 60, $120 \mathrm{~min}$ after glucose load. Plasma glucose and glycosylated hemoglobin $\left(\mathrm{HbA}_{1 \mathrm{c})}\right.$ were soon measured by the glucose-oxidase method and anion exchange HPLC respectively. Plasma samples were frozen and stored at $-70^{\circ} \mathrm{C}$ for the measurements of nesfatin-1, insulin, free fatty acid (FFA) and blood fat levels.

\section{Analytical procedure}

Plasma insulin was measured by radioimmunoassay (RIA) using human insulin as standard (Institute of atomic energy, China). FFA was measured with a commercial kit (Randox Laboratories Ltd, Antrim, UK). Samples for measurement of plasma levels of total cholesterol (TC), high-density lipoprotein cholesterol (HDL-C)and triglycerides (TG) were drawn in lithium-heparin

vacuum tubes and analysed enzymatically using an autoanalyzer (Hitachi 747; Hitachi, Tokyo, Japan). Low-density lipoprotein cholesterol (LDL-C) was calculated using the Friedewald equation $(\mathrm{LDL}-\mathrm{C}(\mathrm{mmol} / \mathrm{l})=$ total cholesterol - HDL-C $-\mathrm{TG} / 2.2)$ (Friedewald et al., 1972).

\section{Plasma nesfatin-1 assay}

Plasma nesfatin-1 levels were measured using a commercially available enzyme-linked immunosorbent assay (ELISA, Phoenix Pharmaceuticals, Belmont, Calif). The linear range of the assay was $0.78-50 \mu \mathrm{g} / \mathrm{L}$. The inter-assay and the intra-assay coefficients of variation were $10 \%$ and $15 \%$, respectively.

\section{Statistical analysis}

Statistical analyses were performed using SPSS 13.0 software (SPSS Inc., Chicago, IL, USA ), and $P<0.05$ (two-tailed) was considered statistically significant. Data was shown as the mean $\pm S D$. We used one-way ANOVA with post hoc (least significant difference) analysis to assess for differences in body composition, anthropometric, metabolic, and hormonal parameters among the T2DM, IGT, and normal controls. Simple and multiple linear regression analyses were used to examine the association between fasting nesfatin-1 levels and other biomarkers. The associations of nesfatin- 1 with IGT and diabetes were examined by multivariate logistic regression analysis that contains: 1 ) nesfatin-1, age and gender; 2) nesfatin-1, age, gender, BMI and WHR; 3 ) nesfatin-1, age, gender, BMI, WHR and lipid profile.

\section{Results}

The clinical characteristics and biochemical results of the 3 groups were given in $\bullet$ Table 1. Patients with nT2DM and IGT had increased levels of fasting blood glucose (FBG), $2 \mathrm{~h}$ blood

Table 1 Clinical characteristic of study groups $(\bar{x} \pm s)$.

\begin{tabular}{|c|c|c|c|}
\hline Group & nT2DM & IGT & NGT \\
\hline n (male/female) & $74(39 / 35)$ & $73(35 / 38)$ & $73(36 / 37)$ \\
\hline age & $54 \pm 11$ & $54 \pm 10$ & $51 \pm 7$ \\
\hline $\operatorname{BMI}\left(\mathrm{kg} / \mathrm{m}^{2}\right)$ & $25.0 \pm 3.7$ & $24.7 \pm 2.7$ & $24.5 \pm 3.6$ \\
\hline waist hip ratio & $0.91 \pm 0.05^{*}$ & $0.90 \pm 0.08$ & $0.87 \pm 0.08$ \\
\hline FBG (mmol) & $10.9 \pm 3.8^{* *} \ddagger$ & $6.0 \pm 0.4^{* *}$ & $5.4 \pm 0.4$ \\
\hline 2h-PBG (mmol/L) & $21.2 \pm 9.7^{* * \ddagger}$ & $8.9 \pm 1.3^{* *}$ & $5.93 \pm 0.81$ \\
\hline FINS (mU/L) & $8.26 \pm 4.25$ & $8.82 \pm 3.05$ & $7.74 \pm 2.85$ \\
\hline 2h-INS (mU/L) & $45.89 \pm 38.52 * \dagger$ & $60.48 \pm 36.97^{* *}$ & $26.37 \pm 16.72$ \\
\hline $\mathrm{HOMA}_{-\mathrm{IR}}$ & $3.79 \pm 1.77^{* * \ddagger}$ & $2.37 \pm 1.06^{* *}$ & $1.85 \pm 0.66$ \\
\hline HOMA-IS $_{-I S}$ & $31.0 \pm 28.0 * * \ddagger$ & $72.2 \pm 35.2^{* *}$ & $88.8 \pm 49.4$ \\
\hline $\mathrm{Hb} \mathrm{A}_{1} \mathrm{c}(\%)$ & $8.8 \pm 2.4^{* * \ddagger}$ & $5.9 \pm 0.4^{* *}$ & $5.5 \pm 0.4$ \\
\hline $\begin{array}{l}\text { triglyceride } \\
(\mathrm{mmol} / \mathrm{L})\end{array}$ & $2.08 \pm 1.56^{* *}$ & $1.63 \pm 1.02$ & $1.34 \pm 0.68$ \\
\hline $\begin{array}{l}\text { total cholesterol } \\
(\mathrm{mmol} / \mathrm{L})\end{array}$ & $5.25 \pm 1.4$ & $4.98 \pm 1.33$ & $4.83 \pm 0.83$ \\
\hline HDL-C (mmol/L) & $1.29 \pm 0.69$ & $1.22 \pm 0.37$ & $1.27 \pm 0.29$ \\
\hline LDL-C (mmol/L) & $3.01 \pm 1.09$ & $2.73 \pm 0.81$ & $2.69 \pm 0.68$ \\
\hline FFA (mmol/L) & $0.68 \pm 0.31$ & $0.73 \pm 0.51$ & $0.66 \pm 0.47$ \\
\hline \multicolumn{4}{|c|}{$\begin{array}{l}\text { * Values are mean } \pm \text { SD or frequency (percent). nT2DM, newly diagnosed type } 2 \\
\text { diabetes mellitus; IGT, impaired glucose regulate; NGT, normal glucose tolerance; } \\
\text { BMI, body mass index; FBG, fasting blood glucose; } 2 \text { h-PBG, } 2 \text { h blood glucose } \\
\text { after a } 75 \text { g glucose load; FFA, free fatty acids; FINS, fasting plasma insulin; } 2 \text { hINS, } \\
2 \text { h plasma insulin after a glucose load; HOMA-IR, HOMA-insulin resistance index; } \\
\text { HOMA-IS, HOMA- } \beta \text { cell secretion index; HDL-C, High-density lipoprotein cholesterol; } \text {, } \\
\text { LDL-C, Low-density lipoprotein cholesterol. }{ }^{*}<0.05,{ }^{*}<0.01 \text { vs. NGT group. }{ }^{\dagger}<0.05 \text {, } \\
\ddagger<0.01 \text { IGT group }\end{array}$} \\
\hline
\end{tabular}


glucose after a $75 \mathrm{~g}$ glucose load (2hPBG), $2 \mathrm{~h}$ plasma insulin after the glucose load (2hINS), $\mathrm{HbA}_{1 \mathrm{C}}, \mathrm{HOMA}_{\mathrm{IR}}$, and decreased levels of HOMA- ${ }_{\text {IS }}$, when compared with the controls $(P<0.05$ or $P<0.01$ Table 1). The WHR and TG in nT2DM patients were also significantly higher than those in the control subjects. Plasma nesfatin-1 levels were significantly increased in both the nT2DM and IGT groups when compared with the controls ( $1.91 \pm 0.79$ and $1.80 \pm 0.80$ vs. $1.41 \pm 0.58 \mu \mathrm{g} / \mathrm{L}$, both $P<0.01$, $\odot$ Fig. 1 ), but there were no significant differences between the nT2DM and IGT groups. After adjusted for differences in BMI, plasma levels of nesfatin- 1 were still higher in the nT2DM and IGT groups than the controls $(1.91 \pm 0.10$ and $1.80 \pm 0.10$ vs. $1.40 \pm 0.10 \mu \mathrm{g} / \mathrm{L}$, both $P<0.01)$. Other parameters, such as BMI, fasting plasma insulin (FINS), TC, HDL-C, LDL-C, FFA, did not dif-

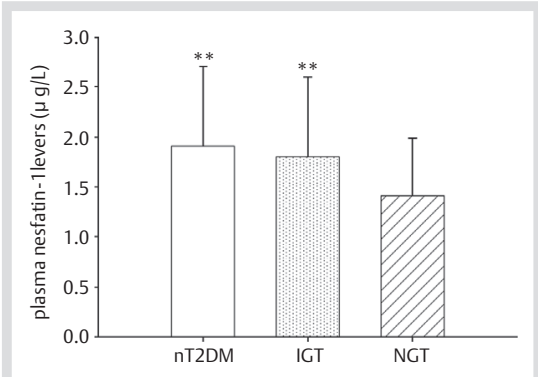

Fig. 1 Plasma nesfatin-1 levels in 3 groups nT2DM, newly type 2 diabetes mellitus; IGT, impaired glucose tolerance; NGT: normal glucose tolerance vs. NGT group ${ }^{* *} P<0.01$.

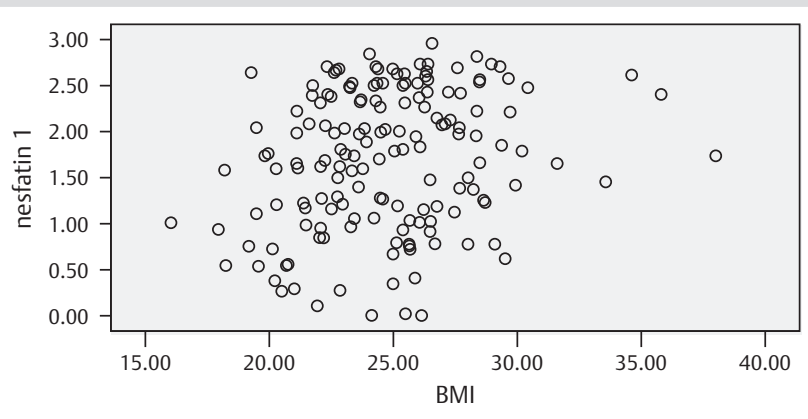

Fig. 2 Correlations of plasma nesfatin- 1 levels with $B M I$ in study subjects $r=0.234, P<0.05$. fer among the different groups. There were no age and gender differences.

We used linear regression analysis to examine the association between plasma nesfatin-1 levels and other metabolic parameters related to insulin resistance. Plasma nesfatin-1 was positively correlated with BMI ( $\bullet$ Fig. 2), $\mathrm{HbA}_{1 \mathrm{C}}, \mathrm{FBG}, 2 \mathrm{hPBG}$, FINS and $\mathrm{HOMA}_{-\mathrm{IR}}$ with simple regression analysis using the pooled data ( Table 2). After adjustment for BMI, plasma nesfatin-1 remained positively correlated with $\mathrm{HbA}_{1 \mathrm{C}}(r=0.21, P<0.01)$, FBG $(r=0.24, P<0.01), 2$ hPBG $(r=0.27, P<0.01)$, FINS $(r=0.16$, $P<0.05)$ and HOMA- $_{\text {IR }}(r=0.28, P<0.01)$. On the other hand, with multiple stepwise regression analysis, only HOMA-IR and BMI remained independently correlated with plasma nesfatin-1 levels ( $($ Table 2 ). The multiple regression equation was: $\left(\mathrm{Y}=0.142 \mathrm{X}_{\mathrm{HOMA}-\mathrm{IR}}+0.043 \mathrm{X}_{\mathrm{BMI}}+0.269\right)$. Multivariate logistic regression analysis showed that plasma nesfatin-1 was significantly associated with IGT and T2DM even after controlling for anthropometric variables and lipid profile ( $\bullet$ Table 3 ).

\section{Discussion \\ $\nabla$}

In the present study, we demonstrated that when compared to control subjects, plasma nesfatin-1 levels were elevated both in patients with nT2DM and with IGT. These results were consistent with a similar observation showing that circulating and adipose tissue protein levels of nesfatin-1 were higher in diet-induced obese mice (Ramanjaneya et al., 2010). Type 2 diabetes mellitus is a genetically heterogeneous disorder and is associated with insulin resistance and impaired insulin secretion (Taylor et al., 1994). Its incidence is rising, in parallel with the growing epidemic of obesity. Nesfatin-1 is a newly discovered physiological regulator of food intake. When injected into the third brain ventricle, nesfatin- 1 significantly decreased food intake and body weight in rats (Oh-I et al., 2006; Johnstone et al., 2006; Stengel et al., 2009). The physiological consequences of elevated plasma nesfatin-1 in patients with nT2DM or IGT found in present study are uncertain. Via its putative anoretic action, it may result in reduced body fat, and improved insulin sensitivity. However, the roles of nesfatin-1 in the pathogenesis of insulin resistance and T2DM are presently not well understood.

\begin{tabular}{|c|c|c|c|c|c|}
\hline \multirow[t]{2}{*}{ Variable } & \multicolumn{2}{|c|}{ Simple } & \multicolumn{2}{|c|}{ Multiple } & \multirow[b]{2}{*}{$95 \% \mathrm{Cls}$} \\
\hline & Estimate & P-value & Estimate & P-value & \\
\hline age & 0.62 & 0.434 & & - & - \\
\hline body mass index & & & 0.043 & 0.013 & $0.063-0.213$ \\
\hline waist-to-hip ratio & 0.044 & 0.581 & & - & - \\
\hline triglyceride & 0.141 & 0.076 & & - & - \\
\hline total cholesterol & 0.008 & 0.920 & & - & - \\
\hline HDL & 0.099 & 0.214 & & - & - \\
\hline LDL & -0.060 & 0.453 & & - & - \\
\hline free fatty acids & 0.032 & 0.689 & & - & - \\
\hline hemoglobin $A_{1} C$ & 0.177 & 0.025 & & - & - \\
\hline FBG & 0.206 & 0.009 & & & - \\
\hline 2h-PBG & 0.248 & 0.002 & & - & - \\
\hline fasting plasma insulin & 0.213 & 0.007 & & - & - \\
\hline 2hINS & 0.103 & 0.201 & & - & - \\
\hline $\mathrm{HOMA}_{\mathrm{IR}}$ & 0.309 & 0.000 & 0.142 & 0.000 & $0.011-0.077$ \\
\hline $\mathrm{HOMA}_{1 \mathrm{~S}}$ & -0.036 & 0.654 & - & & - \\
\hline
\end{tabular}

Table 2 The results of linear regression analysis of variables associated with plasma nesfatin-1 levels in study subjects.

2h-PBG, $2 \mathrm{~h}$ blood glucose after a $75 \mathrm{~g}$ glucose load; $2 \mathrm{hINS}, 2 \mathrm{~h}$ plasma insulin after the glucose load; HOMA $\mathrm{IR}_{\mathrm{R}}$, HOMA-insulin resistance; HOMA-IS, HOMA- $\beta$ cell secretion index; HDL-C, high-density lipoprotein cholesterol; LDL-C, low-density lipoprotein cholesterol 
Table 3 Association of plasma nesfatin-1 with IGT and Type 2 diabetes in fully adjusted models.

\begin{tabular}{|c|c|c|c|c|c|c|}
\hline \multirow[t]{2}{*}{ Model adjusted for } & \multicolumn{3}{|c|}{ IGT } & \multicolumn{3}{|c|}{ nT2DM } \\
\hline & OR & $95 \% \mathrm{Cl}$ & P-value & OR & $95 \% \mathrm{Cl}$ & P-value \\
\hline age, gender & 0.037 & $0.004-0.326$ & 0.003 & 0.052 & $0.006-0.442$ & 0.007 \\
\hline age, gender, BMI, WHR & 0.023 & $0.002-0.220$ & 0.001 & 0.003 & $0.004-0.309$ & 0.003 \\
\hline age, gender, BMI, WHR TG, TC, HDL-C, LDL-C, FFA & 0.019 & $0.002-0.199$ & 0.001 & 0.032 & $0.003-0.331$ & 0.004 \\
\hline
\end{tabular}

nT2DM, newly type 2 diabetes mellitus; IGT, impaired glucose tolerance; NGT: normal glucose tolerance; BMI, body mass index; WHR, waist hip ratio; TG, triglyceride; TC, total cholesterol; HDL-C, high-density lipoprotein cholesterol; LDL-C, low-density lipoprotein cholesterol; FFA, free fatty acids; OR: odds ratio

The another report on plasma nesfatin-1 levels in humans showed that it was lower in T2DM patients compared with controls (Li et al., 2010). Differences in study design, including patient selection (e.g., obese vs. lean, glycemia level, diet type) and experimental conditions, may have contributed to the discrepancy between these results and ours. For instance, Li QC et al. (Li et al., 2010) studied patients at various stages of the disease, some of whom had macrovascular and microvascular complications and were on multiple medications including oral hypoglycemic drugs and insulin, all of which could affect the release of nesfatin-1 (Gonzalez et al., 2009). These may thus be limiting the power of the analysis. In comparison, in the present study, patients were newly diagnosed, were not treated with oral hypoglycemic agents, nor were on special diets. A previous study in vitro had shown that the release of NUCB2/nesfatin-1 from isolated rat islets significantly increased in response to glucose (Foo et al., 2010). Thus it is possible that $\beta$-cells secrete normal amounts of nesfatin- 1 and insulin only during the early stages of diabetes. Hence, we designed this study based on excluding the effects of macrovascular and microvascular complications and multiple medications on plasma nesfatin-1.

With simple regression analysis, plasma nesfatin-1 levels were significantly correlated with BMI, which was in agreement with recent studies (Ramanjaneya et al., 2010; Tan et al., 2011; Ogiso et al., 2011), but in disagreement with another study (Aydin et al., 2009). We studied patients with nT2DM, who differed from the studies mentioned above. As a novel adipokine, nesfatin-1 is increased in obese states in both rodents and humans (Ramanjaneya et al., 2010). For instance, Tan et al. studied subjects consisted of Caucasian (14 overweight, BMI, 25.0 to $<30 \mathrm{~kg} / \mathrm{m}^{2} ; 10$ obese, BMI, $\left.\geq 30 \mathrm{~kg} / \mathrm{m}^{2}\right)$, who differed from our studies with respect to BMI (74 nT2MD, BMI, $25.0 \pm 3.7 \mathrm{~kg} / \mathrm{m}^{2} ; 73$ IGT, BMI, $24.7 \pm 2.7 \mathrm{~kg} / \mathrm{m}^{2}$ ) (Tan et al., 2011). Thus, ethnicity and patient selection (e.g., Caucasian vs. Chinese; obese vs. lean; glycemia level) may explain the different levels of nesfatin-1 reported by Tan et al. (Tan et al., 2011). In addition, the differences of study designs and experimental conditions likely contribute to the disparity. However, as with most new discoveries, these findings need to be reproduced.

Simple regression analysis demonstrated that plasma nesfatin-1 level was also positively correlated with $\mathrm{HbA}_{1 \mathrm{c}}$, FBG, 2hPBG, FINS and $\mathrm{HOMA}_{\mathrm{IR}}$. Usually, increased plasma levels of $\mathrm{HbA}_{1 \mathrm{C}}, \mathrm{FBG}$, $2 \mathrm{hPBG}$ and FINS were caused by insulin resistance, and aggravated T2DM. In multiple stepwise regression analysis, only HOMA- $_{\text {IR }}$ and BMI were independently related factors with plasma nesfatin-1 levels. Therefore, a possible reason for the increased levels of nesfatin-1 in nT2DM patients might be insulin resistance, as has been suggested by a previous study (Ramanjaneya et al., 2010).

Our study was limited by its cross-sectional design, a relatively small sample size and therefore did not prove a causal relation- ship between increased plasma nesfatin-1 levels and the development of T2DM.

In conclusion, the novelty of our study is that we demonstrate, for the first time, elevated nesfatin-1 levels in nT2DM and IGT. Furthermore, plasma nesfatin-1 levels are associated with several metabolic and anthropometric parameters in nT2DM and IGT subjects. Association with these metabolic indices suggests that elevated nesfatin-1 levels may be an anorexigenic factor that opposes weight gain and improves insulin resistance.

\section{Acknowledgements \\ $\nabla$}

This work was supported by grants from the National Natural Science Foundation of China (30871199, 30771037, 30971388 and 81070640), and the Doctoral Fund of the Ministry of Education of China (20105503110002).

Conflict of Interest: None of the authors have a conflict of interest.

\section{Affiliations}

'Department of Endocrinology, the Second Affiliated Hospital, Chongqing Medical University, Chongqing, China

${ }^{2}$ The Key Laboratory of Laboratory Medical Diagnostics in the Ministry of Education and Department of Clinical Biochemistry, Chongqing Medical University College of Laboratory Medicine, Chongqing, China

${ }^{3}$ Department of Pediatrics, University of Mississippi Medical Center, Jackson, Mississippi, USA

${ }^{4}$ The Division of Endocrinology/Diabetes/Metabolism and the Clinical Research Center, Temple University School of Medicine, Philadelphia, Pennsylvania, USA

\section{References}

1 Oh-I S, Shimizu H, Satoh T et al. Identification of nesfatin-1 as a satiety molecule in the hypothalamus. Nature 2006; 443: 709-712

2 Johnstone LE, Fong TM, Leng G. Neuronal activation in the hypothalamus and brainstem during feeding in rats. Cell Metab 2006; 4 : 257-258

3 Stengel A, Goebel M, Wang $L$ et al. Central nesfatin-1 reduces darkphase food intake and gastric emptying in rats: differential role of corticotropin-releasing factor2 receptor. Endocrinology 2009; 150: 4911-4919

4 Shimizu H, Oh-I S, Hashimoto $K$ et al. Peripheral administration of nesfatin-1 reduces food intake in mice: the leptin-independent mechanism. Endocrinology 2009; 150: 662-671

5 Kohno D, Nakata M, Maejima Y et al. Nesfatin-1 neurons in paraventricular and supraoptic nuclei of the rat hypothalamus coexpress oxytocin and vasopressin and are activated by refeeding. Endocrinology 2008; 149: 1295-1301

6 Stengel A, Goebel M, Yakubov I et al. Identification and characterization of nesfatin-1 immunoreactivity in endocrine cell types of the rat gastric oxyntic mucosa. Endocrinology 2009; 150: 232-238

7 Goebel M, Stengel A, Wang L et al. Nesfatin-1 immunoreactivity in rat brain and spinal cord autonomic nuclei. Neurosci Lett 2009; 452: 241-246 
8 Foo KS, Brauner H, Ostenson CG et al. Nucleobindin-2/nesfatin in the endocrine pancreas: distribution and relationship to glycaemic state. J Endocrinol 2010; 204: 255-263

9 Rasouli N, Kern PA. Adipocytokines and the metabolic complications of obesity. J Clin Enodocrinol Metab 2008; 93: S64-S73

10 Alberti KG, Zimmet PZ. Definition, diagnosis and classification of diabetes mellitus and its complications. Part 1: diagnosis and classification of diabetes mellitus, provisional report of a WHO consultation. Diabetic Med 1998; 15: 539-553

11 Albareda M, Rodriguez-Espinosi J, Murugo M et al. Assessment of insulin sensitivity and beta-cell function from measurements in the fasting state and during an oral glucose tolerance test. Diabetologia 2000; 43: $1507-1511$

12 Friedewald WT, Levy RI, Frederickson DS. Estimation of the concentration of low-density lipoprotein cholesterol in plasma, without use of the preparative ultracentrifugation. Clin Chem 1972; 8: 499-502

13 Ramanjaneya $M$, Chen J, Brown JE et al. Identification of nesfatin-1 in human and murine adipose tissue: A novel depot-specific adipokine with increased levels in obesity. Endocrinology 2010; 151: $1358-1363$
14 Taylor SI, Accili D, Imai Y. Insulin resistance or insulin deficiency. Which is the primary cause of NIDDM? Diabetes 1994; 43: 735-740

15 Li QC, Wang HY, Chen $X$ et al. Fasting plasma levels of nesfatin-1 in patients with type 1 and type 2 diabetes mellitus and the nutrientrelated fluctuation of nesfatin-1 level in normal humans. Regul Pept 2010; 159: 72-77

16 Gonzalez R, Tiwari A, Unniappan S. Pancreatic beta cells colocalize insulin and pronesfatin immunoreactivity in rodents. Bio Bio Res Commun 2009; 381: 643-648

17 Tan BK, Hallschmid M, Kern $W$ et al. Decreased cerebrospinal fluid/ plasma ratio of the novel satiety molecule, nesfatin-1/nucb-2, in obese human: evidence of nesfatin-1/nucb-2 resistance and implications for obesity treatment. JCEM 2011; 96: E669-E673

18 Ogiso K, Asakawa A, Amitani $\mathrm{H}$ et al. Plasma nesfatin-1 concentration in restricting-type anorexia nervosa. Peptide 2011; 32: 150-153

19 Aydin S, Dag E, Ozkan Y et al. Nesfatin-1and ghrelin levels in serum and saliva of epileptic patients: hormonal changes can have a major effect on seizure disorders. Mol Cell Biochem 2009; 328: 49-56 